\title{
Histological evaluations of primary lesions are independently associated with prognosis in patients with gastric cancer who receive neoadjuvant chemotherapy
}

\author{
TOMOMITSU TAHARA, TOMOYUKI SHIBATA, MASAAKI OKUBO, DAI YOSHIDA, \\ TOMOHIKO KAWAMURA, NORIYUKI HORIGUCHI, TAKAMITSU ISHIZUKA, \\ MITSUO NAGASAKA, YOSHIHITO NAKAGAWA and NAOKI OHMIYA
}

Department of Gastroenterology, Fujita Health University School of Medicine, Toyoake, Aichi 470-1192, Japan

Received July 1, 2016; Accepted February 23, 2017

DOI: $10.3892 / \mathrm{ol} .2017 .6040$

\begin{abstract}
Neoadjuvant chemotherapy may improve outcomes for patients with locally advanced gastric cancer (GC). To explore useful predictive factors for the response of advanced GC to neoadjuvant chemotherapy, tumor responses were assessed using computed tomography (CT) with histological based criteria. A total of 78 patients with advanced GC undergoing neoadjuvant chemotherapy were included. CT-based response assessment was performed following 2 courses of treatment. Histological evaluation of resected specimens was also performed according to the Japanese classification of gastric carcinoma. Grade 1b, 2 and 3 (viable tumor cells remaining in $<2 / 3$ of the tumorous area) were defined as histological responders. The results were associated with overall survival (OS) and progression-free survival (PFS). The majority of the cases underwent tegafur/gimeracil/oteracil based preoperative chemotherapy as the first line of treatment $(n=76,96 \%)$. A total of $25(32 \%)$ and $29(37 \%)$ cases were considered to be $\mathrm{CT}$ and histological responders, respectively. CT-based evaluation was not associated with OS or PFS, while histological evaluation was significantly associated with OS and PFS. Histological based evaluation was not associated with CT and GI X-ray or endoscopy-based evaluation of primary lesions. Multivariate survival analysis using Cox's regression model demonstrated that histological non-response was an independent prognostic factor for predicting worse OS. Histological-based evaluation of primary lesions was
\end{abstract}

Correspondence to: $\mathrm{Dr}$ Tomomitsu Tahara, Department of Gastroenterology, Fujita Health University School of Medicine, 1-98 Dengakugakubo, Toyoake, Aichi 470-1192, Japan

E-mail: tomomiccyu@yahoo.co.jp

Abbreviations: GC, gastric cancer; CT, computed tomography; OS, overall survival; PFS, progression-free survival

Key words: gastric cancer, neoadjuvant chemotherapy, histological evaluation, overall survival progression-free survival independently associated with prognosis in patients with GC who underwent neoadjuvant chemotherapy.

\section{Introduction}

Gastric cancer (GC) is one of the most common malignancies globally, accounting for $\sim 70,000$ new cases and 650,000 mortalities per year $(1,2)$. Despite advances in strategies for early detection, the majority of patients still present with an advanced disease at diagnosis. The prognosis of patients with advanced tumor remains poor (3). Further decreases in mortality rates would require improved treatment outcomes in patients with advanced GC.

Chemotherapy is recognized as the most effective treatment for patients with unresectable advanced or metastatic GC. To date, multiple clinical trials have evaluated its efficacy and safety (4-9). As well as for unresectable cases, neoadjuvant chemotherapy may also be considered for potentially resectable cases to further improve their outcomes. Several previous studies have evaluated the potential usefulness of neoadjuvant chemotherapy in locally advanced GC (10-14).

Regarding the response assessment to the chemotherapy, an early evaluation during neoadjuvant chemotherapy would be of interest for tailoring chemotherapy based on the individual response. Correct identification of responding or non-responding patients would be important for more appropriate implementation, to avoid toxic and ineffective chemotherapy (15-17).

Histopathological classification of regression is considered to be standard criteria for response assessments to the chemotherapy in GC. The Japanese classification of gastric carcinoma (JCGC) defined histological classification of resected specimens for an early response evaluation of neoadjuvant chemotherapy (18). Previous studies have evaluated the validity of histological evaluation of resected specimens from patients with advanced GC receiving neoadjuvant chemotherapy $(19,20)$. Kurokawa et al (19) compared JCGC histological-based evaluation with response evaluation criteria in solid tumors (RECIST) as well as upper gastrointestinal (GI) X-ray or endoscopy based response evaluation of primary lesions, using two different cohorts. The 
results demonstrated the superiority of histological evaluation compared with RECIST and upper GI X-ray or endoscopy based response evaluation. Heger et al (21) performed histological based evaluations using the scoring systems of Becker et al (20). They also performed computed tomography (CT) and endoscopy-based response evaluation and confirmed a good correlation among the three evaluation systems (21).

To evaluate the validity of JCGC histological classification for an early response evaluation of neoadjuvant chemotherapy in advanced GC, the JCGC histological based evaluation was compared with CT-based response evaluation following 2 courses of chemotherapy. The results demonstrated that histological-based evaluation was superior to the CT-based response evaluation as an independent prognostic predictor in advanced GC being treated with neoadjuvant chemotherapy.

\section{Patients and methods}

Patients, survival and response evaluation using different criteria. The studied population comprised of 78 Japanese patients with advanced $\mathrm{GC}$, receiving neoadjuvant chemotherapy from April 2003 to September 2012 at the Fujita Health University hospital (Toyoake, Japan) All GC cases were diagnosed histologically and were classified according to Lauren's classification (22). Detailed information concerning anatomical location, macroscopic types, depth, lymph node and other metastasis and peritoneal dissemination was obtained according to the JCGC (18).

Using CT, the response to chemotherapy was assessed following 2 courses of treatment (7-10 weeks following initial administration, which varied across the different regimens). If measurable lesions existed, RECIST was applied and cases were classified into complete response (CR), partial response (PR), stable disease (SD) and progressive disease (PD) (23). $\mathrm{CR}$ and $\mathrm{PR}$ were considered to be responders according to RECIST. If RECIST was not applicable, responders were defined as cases with a clear reduction of the primary lesion in the CT images assessed by experienced physicians with the consensus was taken as the final result. Information concerning the upper GI X-ray or endoscopy-based response evaluation of primary lesions was also available for all patients. Upper GI $\mathrm{X}$-ray or endoscopy-based responders were defined as PR or $\mathrm{CR}$ in the JCGC criteria (18). The assessment was performed by experienced physicians and the consensus was taken as the final result. Those who were not considered to be responders by CT and upper GI X-ray or endoscopy-based evaluations were considered to be CT and upper GI X-ray or endoscopy based non-responders, respectively.

All patients underwent gastrectomy with a D2 lymph node dissection following 2 courses of chemotherapy. Histological-based response evaluation of resected tumors was performed by the senior pathologists at the Fujita Health University hospital using Japanese Gastric Cancer Association criteria (18), and all cases were classified as Grade 0, 1a, 1b, 2 or 3. Patients were scored as Grade 0 if there was no evidence of chemotherapeutic effect. Patients were scored as Grade 1a if viable tumor cells remained in $<2 / 3$ of the tumorous area. Patients were scored as Grade $1 \mathrm{~b}$ if viable tumor cells remained in $>1 / 3$ but $<2 / 3$ of the tumorous area. Patients were scored as Grade 2 if viable tumor cells
Table I. Clinicopathological features of patients with gastric cancer.

\begin{tabular}{|c|c|}
\hline Clinicopathological feature & Value \\
\hline Number of patients & 78 \\
\hline Median age (range) & $68(39-83)$ \\
\hline \multicolumn{2}{|l|}{ Gender } \\
\hline Female n (\%) & $21(26.9)$ \\
\hline Male n (\%) & $57(73.1)$ \\
\hline \multicolumn{2}{|l|}{ Location } \\
\hline Upper n (\%) & $18(23.1)$ \\
\hline Middle n (\%) & $35(44.9)$ \\
\hline Lower n (\%) & $25(32.1)$ \\
\hline \multicolumn{2}{|l|}{ Histology } \\
\hline Intestinal n (\%) & $35(44.9)$ \\
\hline Diffuse n (\%) & $35(44.9)$ \\
\hline Mixed n (\%) & $8(10.2)$ \\
\hline \multicolumn{2}{|l|}{ Morphology } \\
\hline Type1 n (\%) & $4(5.1)$ \\
\hline Type2 n (\%) & $25(32.1)$ \\
\hline Type3 n (\%) & $44(56.4)$ \\
\hline Type4 n (\%) & $5(6.4)$ \\
\hline \multicolumn{2}{|l|}{ Staging } \\
\hline II n (\%) & $33(42.3)$ \\
\hline III n (\%) & $34(43.6)$ \\
\hline IV n (\%) & $11(14.1)$ \\
\hline \multicolumn{2}{|l|}{ Depth } \\
\hline $\mathrm{T} 2 \mathrm{n}(\%)$ & $17(21.8)$ \\
\hline $\mathrm{T} 3 \mathrm{n}(\%)$ & $16(20.5)$ \\
\hline T4 n (\%) & $45(57.7)$ \\
\hline \multicolumn{2}{|l|}{ Lymph node metastasis } \\
\hline N0 n $(\%)$ & $23(29.5)$ \\
\hline N1 n $(\%)$ & $17(21.8)$ \\
\hline N2 n $(\%)$ & $20(25.6)$ \\
\hline N3 n $(\%)$ & $18(23.1)$ \\
\hline \multicolumn{2}{|l|}{ Distant metastasis } \\
\hline Peritoneal dissemination $\mathrm{n}(\%)$ & $8(10.3)$ \\
\hline Liver metastasis n (\%) & $3(3.8)$ \\
\hline Other metastasis n (\%) & $2(2.6)$ \\
\hline
\end{tabular}

remained in $<1 / 3$ of the tumorous area. Patients were scored as Grade 3 if no viable tumor cells remained in the section where the tumor was thought to have been located at the pretreatment assessment (18). The evaluation was performed using multiple sections of hematoxylin and eosin staining of paraffin-embedded sections $(4 \mu \mathrm{m})$ of the resected specimen to avoid the influence of tumor heterogeneity. Based on this histological assessment, Grade 1b, 2 and 3 (viable tumor cells remaining in $<2 / 3$ of the tumorous area) cases were defined as histological responders, and all others were considered to be histological non-responders. Overall survival (OS) was defined as the time from the start of initial administration of chemotherapy to the date of cancer-associated mortality. 
Table II. Information concerning the treatment of gastric cancer.

\begin{tabular}{lc}
\hline Variable & $\mathrm{n}(\%)$ \\
\hline Agent & $71(91.0)$ \\
S-1+CDDP & $4(5.1)$ \\
S-1 & $3(3.8)$ \\
Others & \\
Response to chemotherapy $(\mathrm{CT})^{\mathrm{a}}$ & $25(32.5)$ \\
Responder & $52(67.5)$ \\
Non-responder & \\
Response to chemotherapy & \\
(upper GI X-ray or endoscopy) & $53(67.9)$ \\
Responder & $25(32.1)$ \\
Non-responder & \\
Response to chemotherapy & \\
(histological grade) & $2(2.6)$ \\
0 & $47(60.3)$ \\
1a & $16(20.5)$ \\
$1 \mathrm{~b}$ & $11(14.1)$ \\
$2 \mathrm{n}$ & $2(2.6)$ \\
$3 \mathrm{n}$ &
\end{tabular}

anformation was not available for 1 patient. S-1, tegafur/gimeracil/ oteracil; CDDP, cisplatin; CT, computed tomography; GI, gastrointestinal.

Table III. $\kappa$ coefficient value assessing the concordance among histological grade, CT and upper GI X-ray or endoscopy.

\begin{tabular}{lc}
\hline Variable & $\kappa$ coefficient value \\
\hline Histological grade vs. CT & 0.13 \\
Histological grade vs. upper & \\
GI X-ray or endoscopy & 0.17 \\
\hline
\end{tabular}

CT, computed tomography; GI, gastrointestinal.

If cancer-associated mortality had not occurred, the OS was censored on the last date the patient was known to be alive. Progression-free survival (PFS) was defined as the time of initial administration of chemotherapy to tumor progression or cancer-associated mortality. Patients with no confirmation of progression or cancer-associated mortality were censored at the date of the last objective tumor assessment. The Ethical Review Board of the Fujita Health University School of Medicine (Toyoake, Japan) approved the protocol, and written informed consent was obtained from all participating subjects.

Statistical analysis. Categorical variables among the two groups were assessed using the two-tailed Fisher's exact test. A $\kappa$ coefficient value was calculated to assess the consistency of different response evaluations to chemotherapy. OS and PFS among the two groups were assessed using the Kaplan-Meier
Table IV. Multivariate survival analysis using Cox's regression model for adjustment of clinicopathological factors.

\begin{tabular}{lccc}
\hline Variable & HR $(95 \% \mathrm{CI})$ & P-value & C index \\
\hline $\begin{array}{l}\text { Histological grade } \\
\text { (non-responder) }\end{array}$ & $3.97(1.31-11.99)$ & 0.015 & 0.65 \\
$\begin{array}{l}\text { Upper GI X-ray } \\
\text { or endoscopy } \\
\text { (non-responder) }\end{array}$ & $10.10(3.68-27.73)$ & $<0.0001$ & 0.73 \\
$\begin{array}{l}\text { Histology } \\
\text { (intestinal type) }\end{array}$ & $0.30(0.09-0.997)$ & 0.049 & 0.59 \\
\hline
\end{tabular}

GI, gastrointestinal; HR, hazard ratio; CI, confidence intervals.

method and the Log rank test. $\mathrm{P}<0.05$ was considered to indicate a statistically significant difference. Multivariate survival analysis using Cox's regression model was also performed for calculating hazard ratios (HR), 95\% confidence intervals (CI) and a $\mathrm{C}$ index with adjustment for clinicopathological factors.

\section{Results}

Clinicopathological characteristics of subjects and information about the treatment are listed in Table I and II, respectively. The majority of the cases underwent tegafur/ gimeracil/oteracil based preoperative chemotherapy as the first line treatment $(n=76 ; 96 \%)$. OS and PFS were assessed among all cases. The median OS and median PFS in all cases were 34.0 and 20.3 months, respectively. Although all patients were considered to be operable following two courses of chemotherapy, distant metastatic lesions were identified in 13 cases during surgery (Table I).

Response rates using three different criteria are listed in Table II. A total of $25(32 \%)$ and $53(68 \%)$ cases were considered to be CT and upper GI X-ray or endoscopy based responders, respectively. Concerning histological grade, 2 (2.6\%), 47 (60.3\%), 16 (20.5\%), 11 (14.1\%), and 2 (2.6\%) cases were considered to be Grade $0,1 \mathrm{a}, 1 \mathrm{~b}, 2$ and 3, respectively. A total of 29 cases (37\%) were considered to be histological responders (Table II). The $\kappa$ coefficient values were initially calculated to assess the consistency of histological evaluations and the other two criteria. It was demonstrated that there were low consistencies among the histological evaluation and CT $(\kappa=0.13$; Table III) or the histological evaluation and upper GI X-ray or endoscopy ( $\kappa=0.17$; Table III). CT-based evaluation was not significantly associated with $\mathrm{OS}(\mathrm{P}=0.22)$ or PFS $(\mathrm{P}=0.35)$ by the log-rank test. On the other hand, histological evaluation was significantly associated with OS and PFS ( $\mathrm{P}=0.0028$ and $\mathrm{P}=0.0003$, respectively) by the log-rank test (Fig. 1).

To evaluate the independent prognostic factors associated with OS, multivariate survival analysis using Cox's regression model was performed. For this analysis, all clinicopathological factors including sex, age, anatomical location, macroscopic and histologic types, depth, information about metastasis, staging and response to treatment were included. This analysis demonstrated that histological non-response 
OS (CT)

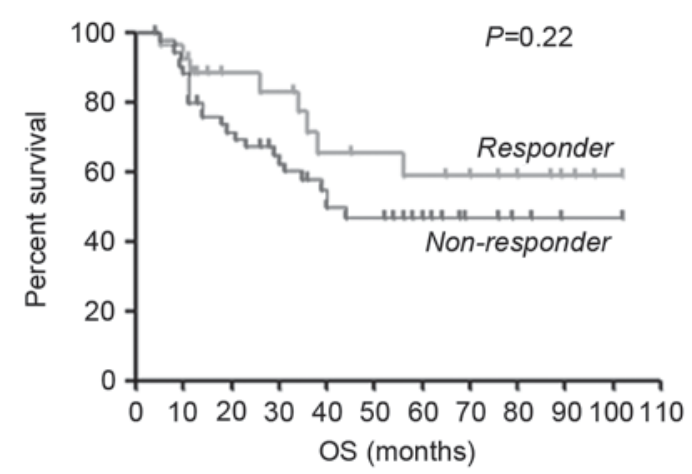

PFS (CT)

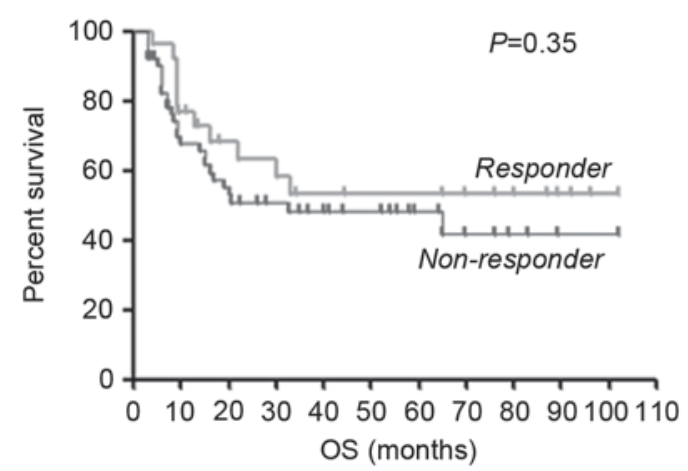

OS (histological evaluation)

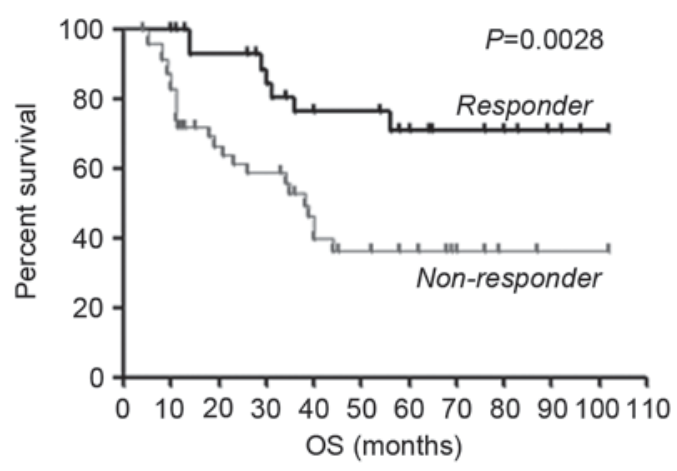

PFS (histological evaluation)

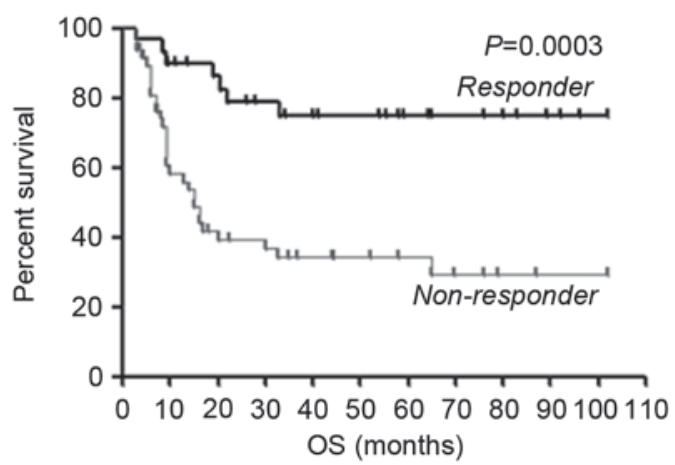

Figure 1. OS and PFS for CT and histological- based response evaluations. The differences between the groups were assessed using the Kaplan-Meier method and the Log rank test. OS, overall survival; PFS, progression free survival; CT, computed tomography.

$(\mathrm{HR}=3.97,95 \% \mathrm{CI}=1.31-11.99, \mathrm{C}$ index=0.65; Table IV $)$ and upper GI X-ray or endoscopy based non-response $(\mathrm{HR}=10.1$, 95\% CI=3.68-27.73, C index=0.73; Table IV) were independent prognostic factors for predicting worse OS, while intestinal histology was associated with improved OS (HR=0.30, 95\% CI=0.09-0.997, $\mathrm{C}$ index=0.59; Table IV). No other factors were demonstrated to be associated with OS by this analysis (data not shown).

\section{Discussion}

The present study has demonstrated that the results of histological based evaluation are a good prognostic predictor for advanced $\mathrm{GC}$ receiving neoadjuvant chemotherapy. RECIST is the most widely accepted criteria for evaluating the response to chemotherapy, but it requires the presence of a measurable lesion. In RECIST, primary gastric tumors are regarded as non-target lesions (23). Since resectable GC usually does not have a measurable lesion, it may be difficult to apply RECIST, in particular for cases receiving neoadjuvant chemotherapy. In the present study, for the cases in which RECIST were not applicable, clear reduction of primary or metastatic lesions in the images of CT were considered to be responders. The results demonstrated that histological based evaluation was superior to CT-based response evaluation for the prediction of prognosis, by univariate and multivariate analysis. These results suggested that CT-based evaluation may not effectively assess the response of locally advanced GC to neoadjuvant chemotherapy, and that histological based evaluation of primary tumor may be more suitable for a precise response assessment of neoadjuvant chemotherapy. The prognostic influence of histological response has been demonstrated in locally advanced GC undergoing neoadjuvant chemotherapy (19). Heger et al (21) demonstrated a correlation between histological, CT and endoscopy based response evaluations. In the results of the present study, however, there were low consistencies among these evaluations. The differences observed in these results may be due to patient constitution and the different time points selected for response evaluation. The response assessment of CT and endoscopy were earlier in the study of Heger et al (21) than the present study.

Multivariate survival analysis demonstrated that histological-based non-response was an independent prognostic factor for predicting worse OS. However, the association of upper GI X-ray or endoscopy based non-responders was stronger, which was different to the results of a previous study. Kurokawa et al (19) compared JCGC histological-based evaluation with RECIST as well as GI X-ray or endoscopy based evaluation. The results demonstrated the superiority of histological evaluation compared with the other two evaluations. However, two different cohorts were investigated by Kurokawa et al (19), thus it was not possible to assess the direct correlation of the three evaluations. 
The low consistency among GI X-ray or endoscopy and histology indicated that objective changes judged in these evaluations may be different. For example, changes observed during GI X-ray or endoscopy may be early morphological changes, as indicated by a decrease in metabolic activity $(16,24)$ or transient tissue reactions, which were less compared with the resected specimens. However, it is also expected that histological response evaluation provides important information since it directly evaluates the influence of chemotherapy on cancer cells.

As histological evaluation is less invasive and more objective compared with GI X-ray or endoscopy, it may be recommended for all cases. Precise assessment of responses to chemotherapy would be of great interest for tailoring chemotherapy based on the individual response. As histological response was associated with long-term outcomes of patients with GC, it would be useful for identification of responding or non-responding patients to avoid toxic and ineffective chemotherapy (15-17).

\section{References}

1. Ferlay J, Bray F, Parkin DM and Pisani P (eds): Gobocan 2000 Cancer incidence and mortality worldwide (IARC Cancer Bases No. 5). Lyon: IARC Press, 2001.

2. Lau M, Le A and El-Serag HB: Noncardia gastric adenocarcinoma remains an important and deadly cancer in the United States: Secular trends in incidence and survival. Am J Gastroenterol 101: 2485-2492, 2006.

3. Nashimoto A, Akazawa K, Isobe Y, Miyashiro I, Katai H, Kodera Y, Tsujitani S, Seto Y, Furukawa H, Oda I, et al: Gastric cancer treated in 2002 in Japan: 2009 annual report of the JGCA nationwide registry. Gastric Cancer 16: 1-27, 2013.

4. Boku N, Yamamoto S, Fukuda H, Shirao K, Doi T, Sawaki A, Koizumi W, Saito H, Yamaguchi K, Takiuchi H, et al: Fluorouracil versus combination of irinotecan plus cisplatin versus S-1 in metastatic gastric cancer: A randomised phase 3 study. Lancet Oncol 10: 1063-1069, 2009.

5. Yamaguchi K, Sawaki A, Doi T, Satoh T, Yamada Y, Omuro Y, Nishina T, Boku N, Chin K, Hamamoto Y, et al: Efficacy and safety of capecitabine plus cisplatin in Japanese patients with advanced or metastatic gastric cancer: Subset analyses of the AVAGAST study and the ToGA study. Gastric Cancer 16: 175-182, 2013.

6. Koizumi W, Nakayama N, Tanabe S, Sasaki T, Higuchi K, Nishimura K, Takagi S, Azuma M, Ae T, Ishido K, et al: A multicenter phase II study of combined chemotherapy with docetaxel, cisplatin and S-1 in patients with unresectable or recurrent gastric cancer (KDOG 0601). Cancer Chemother Pharmacol 69: 407-413, 2012.

7. Koizumi W, Narahara H, Hara T, Takagane A, Akiya T, Takagi M, Miyashita K, Nishizaki T, Kobayashi O, Takiyama W, et al: S-1 plus cisplatin versus S-1 alone for first-line treatment of advanced gastric cancer (SPIRITS trial): A phase III trial. Lancet Oncol 9: 215-21, 2008

8. Okines AF, Norman AR, McCloud P, Kang YK and Cunningham D: Meta-analysis of the REAL-2 and ML17032 trials: Evaluating capecitabine-based combination chemotherapy and infused 5-fluorouracil-based combination chemotherapy for the treatment of advanced oesophago-gastric cancer. Ann Oncol 20: 1529-1534, 2009.

9. Hamaguchi T, Shirao K, Ohtsu A, Hyodo I, Arai Y, Takiuchi H, Fujii H, Yoshida M, Saito H, Denda T, et al: A phase II study of biweekly mitomycin $\mathrm{C}$ and irinotecan combination therapy in patients with fluoropyrimidine-resistant advanced gastric cancer: A report from the Gastrointestinal oncology group of the Japan clinical oncology group (JCOG0109-DI Trial). Gastric Cancer 14: 226-233, 2011.
10. Cunningham D, Allum WH, Stenning SP, Thompson JN, Van de Velde CJ, Nicolson M, Scarffe JH, Lofts FJ, Falk SJ, Iveson TJ, et al: Perioperative chemotherapy versus surgery alone for resectable gastroesophageal cancer. N Engl J Med 355: 11-20, 2006.

11. Kodera Y, Ishiyama A, Yoshikawa T, Kinoshita T, Ito S, Yokoyama H, Mochizuki Y, Ito H, Tsuburaya A, Sakamoto J, et al: A feasibility study of postoperative chemotherapy with S-1 and cisplatin (CDDP) for gastric carcinoma (CCOG0703). Gastric Cancer 13: 197-203, 2010.

12. Tsuburaya A, Nagata N, Cho H, Hirabayashi N, Kobayashi M, Kojima H, Munakata Y, Fukushima R, Kameda Y, Shimoda T, et al: Phase II trial of paclitaxel and cisplatin as neoadjuvant chemotherapy for locally advanced gastric cancer. Cancer Chemother Pharmacol 71: 1309-1314, 2013.

13. Hirakawa M, Sato Y, Ohnuma H, Takayama T, Sagawa T, Nobuoka T, Harada K, Miyamoto H, Sato Y, Takahashi Y, et al: A phase II study of neoadjuvant combination chemotherapy with docetaxel, cisplatin, and S-1 for locally advanced resectable gastric cancer: Nucleotide excision repair (NER) as potential chemoresistance marker. Cancer Chemother Pharmacol 71: 789-797, 2013.

14. Yoshikawa T, Rino Y, Yukawa N, Oshima T, Tsuburaya A and Masuda M: Neoadjuvant chemotherapy for gastric cancer in Japan: A standing position by comparing with adjuvant chemotherapy. Surg Today 44: 11-21, 2014.

15. Ott K, Weber WA, Lordick F, Becker K, Busch R, Herrmann K, Wieder H, Fink U, Schwaiger M and Siewert JR: Metabolic imaging predicts response, survival, and recurrence in adenocarcinomas of the esophagogastric junction. J Clin Oncol 24: 4692-46988, 2006

16. Weber WA, Ott K, Becker K, Dittler HJ, Helmberger H, Avril NE, Meisetschläger G, Busch R, Siewert JR, Schwaiger M and Fink U: Prediction of response to preoperative chemotherapy in adenocarcinomas of the esophagogastric junction by metabolic imaging. J Clin Oncol 19: 3058-3065, 2001.

17. Lordick F, Ott K, Krause BJ, Weber WA, Becker K, Stein HJ, Lorenzen S, Schuster T, Wieder H, Herrmann K, et al: PET to assess early metabolic response and to guide treatment of adenocarcinoma of the oesophagogastric junction: The MUNICON phase II trial. Lancet Oncol 8: 797-805, 2007.

18. Japanese Gastric Cancer Association: Japanese classification of gastric carcinoma: 3rd English edition. Gastric Cancer 14: 101-112, 2011.

19. Kurokawa Y, Shibata T, Sasako M, Sano T, Tsuburaya A, Iwasaki Y and Fukuda H: Validity of response assessment criteria in neoadjuvant chemotherapy for gastric cancer (JCOG0507-A). Gastric Cancer 17: 514-521, 2014.

20. Becker K, Mueller JD, Schulmacher C, Ott K, Fink U, Busch R, Böttcher K, Siewert JR and Höfler H: Histomorphology and grading of regression in gastric carcinoma treated with neoadjuvant chemotherapy. Cancer 98: 1521-1530, 2003.

21. Heger U, Bader F, Lordick F, Burian M, Langer R, Dobritz M, Blank S, Bruckner T, Becker K, Herrmann K, et al: Interim endoscopy results during neoadjuvant therapy for gastric cancer correlate with histopathological response and prognosis. Gastric Cancer 17: 478-488, 2014.

22. Lauren P: The two histological main types of gastric carcinoma: Diffuse and so-called intestinal-type carcinoma. An attempt at a histo-clinical classification. Acta Pathol Microbiol Scand 64: 31-49, 1965

23. Eisenhauer EA, Therasse P, Bogaerts J, Schwartz LH, Sargent D, Ford R, Dancey J, Arbuck S, Gwyther S, Mooney M, et al: New response evaluation criteria in solid tumours: Revised RECIST guideline (version 1.1). Eur J Cancer 45: 228-247, 2009.

24. Ott K, Fink U, Becker K, Stahl A, Dittler HJ, Busch R, Stein H, Lordick F, Link T, Schwaiger M, et al: Prediction of response to preoperative chemotherapy in gastric carcinoma by metabolic imaging: Results of a prospective trial. J Clin Oncol 21: 4604-4610, 2003. 